\title{
Prognostic and predictive values of CDK1 and MAD2L1 in lung adenocarcinoma
}

\author{
Yuan-Xiang Shi' ${ }^{1,2}$, Tao Zhu' ${ }^{1}$, Ting Zou ${ }^{1}$, Wei Zhuo ${ }^{1}$, Yi-Xin Chen ${ }^{1}$, Ma-Sha Huang ${ }^{1}$, \\ Wei Zheng ${ }^{1}$, Chen-Jing Wang ${ }^{1}$, Xi Li ${ }^{1}$, Xiao-Yuan Mao ${ }^{1}$, Wei Zhang ${ }^{1}$, Hong-Hao \\ Zhou $^{1}$, Ji-Ye Yin ${ }^{1,2}$, Zhao-Qian Liu' ${ }^{1,2}$ \\ ${ }^{1}$ Department of Clinical Pharmacology, Xiangya Hospital, Central South University, Changsha 410008, P. R. China; Institute of \\ Clinical Pharmacology, Central South University, Hunan Key Laboratory of Pharmacogenetics, Changsha 410078, P. R. China \\ ${ }^{2}$ Hunan Province Cooperation Innovation Center for Molecular Target New Drug Study, Hengyang 421001, P.R.China \\ Correspondence to: Zhao-Qian Liu, email: liuzhaoqian63@126.com \\ Ji-Ye Yin, email: yinjiye@csu.edu.cn
}

Keywords: prognosis, biomarker, lung cancer, CDK1, MAD2L 1

Received: August 23, 2016

Accepted: October 28, 2016

Published: November 09, 2016

\section{ABSTRACT}

Lung cancer remains as the leading cause of cancer-related death worldwide, and lung adenocarcinoma (LUAD) is the most common histological subtype. This study aims to investigate biomarkers associated with cancer progression and prognosis of LUAD. We integrated expression profiles of 668 lung cancer patients in five datasets from the Gene Expression Omnibus (GEO) and identified a panel of differentially expressed genes (DEGs). Function enrichment analysis highlighted that these genes were closely associated with the carcinogenesis of LUAD, such as cell cycle, ECMreceptor interaction and p53 signaling pathway. Cyclin-dependent kinase 1 (CDK1) and MAD2 mitotic arrest deficient-like 1 (MAD2L1), two critical mitotic checkpoint genes, were selected for further study. Elevated expression of CDK1 and MAD2L1 was validated in an independent LUAD cohort. Kaplan-Meier analysis revealed that CDK1 and MAD2L1 expression was negatively correlated with both overall survival (OS) and relapse-free survival (RFS). In conclusion, CDK1 and MAD2L1 were adverse prognostic biomarkers for LUAD whose increased expression could render patients with LUAD a high risk of cancer recurrence and poor survival, suggesting that they might be applied as potential targets for LUAD treatment.

\section{INTRODUCTION}

Lung cancer is the leading cause of cancer-related death worldwide, of which non-small cell lung cancer (NSCLC) accounts for approximately $85 \%$ cases [1]. NSCLC comprises three major histological subtypes: squamous cell carcinoma (LUSC), adenocarcinoma (LUAD) and large cell carcinoma. The most common type of NSCLC is adenocarcinoma, which comprises around $40 \%$ of all lung cancer [2]. Despite of advances in the diagnosis and treatment for NSCLC, the 5-year survival rate for advanced NSCLC remains poor. It is still urgent to identify sensitive and specific biomarkers that could predict tumor recurrence and prognosis to guide the treatment of NSCLC.

Genome-wide expression profiles have recently been used to identify prognostic signatures in patients with cancer [3-6]. However, some genes identified with prognostic implications in one cohort might be difficult to be verified in other cohorts [7,8]. An explanation might be that the effects of genes with broad confidence intervals are difficult to confirm using a validation strategy, that is, when genes are identified as significant in one study, they are further tested for significance in separate subsequent studies with smaller sample sizes [9]. To address these issues, validation of the signature genes in several independent studies or distinct patient populations is necessary.

In the current study, we compared gene expression changes between tumor tissues and adjacent non-tumor lung tissues (NTL) using five datasets, and overlapped the differentially expressed genes, which could be specifically involved in development of LUAD. We identified 125 differentially expressed genes in LUAD that were 
common among all five profiles. In addition, we validated the overlapping genes in independent patient cohorts. Further, our function enrichment analysis showed that genes related with cell cycle were the most significantly enriched. Thus, in this study, we focused on CDK1 and MAD2L1, two critical mitotic checkpoint genes which play an important role in the mitotic process. We found that CDK1 and MAD2L1 were up-regulated in LUAD and directly correlated with the clinical pathological features. We further investigated and explored the prognostic value of CDK1 and MAD2L1 in LUAD. Our data indicated that high CDK1 or MAD2L1 expression was associated with poor prognosis of LUAD.

\section{RESULTS}

\section{Identification of differentially expressed genes}

In our study, gene expression profiles from five datasets were utilized to compare gene expression between tumors and NTL. Three NSCLC gene expression profiles (GSE19804, GSE19188, GSE18842) and two LUAD gene expression profiles (GSE40791, GSE10072) were analyzed to identify the differentially expressed genes (DEGs) during tumorigenesis. Genes with corrected P-value $<0.05$ and absolute fold change $>2$ were considered as DEGs. The results showed that, 1,388 genes (454 up-regulated and 934 down-regulated genes) were identified to be differentially expressed in GSE19804, 2,421 genes (788 up-regulated and 1,633 down-regulated genes) differentially expressed in GSE19188, 3,168 genes (1,403 up-regulated and 1,765 down-regulated genes) in GSE18842, 3,796 genes (1,646 up-regulated and 2,150 down-regulated genes) in GSE40791 and 666 genes (234 up-regulated and 432 down-regulated genes) in GSE18842 (Figure 1A-1E). Then, we performed an exploratory two-dimensional hierarchical clustering of the differentially expressed probes, the mRNA expression profiles of tumors and NTL resulted in separate clusters (Supplementary Figure S1). Additionally, we conducted an overlapping analysis of the DEGs in NSCLC and LUAD to identify
A

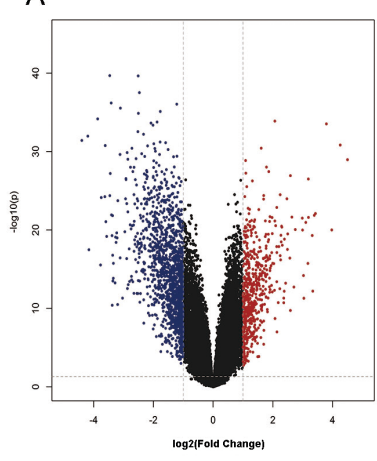

E

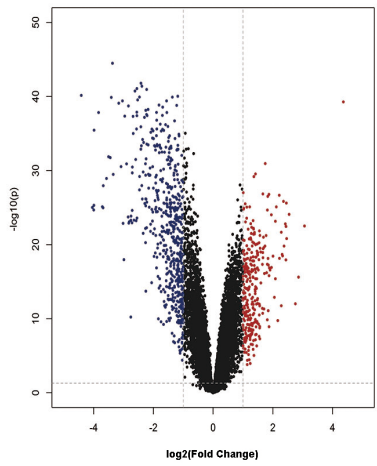

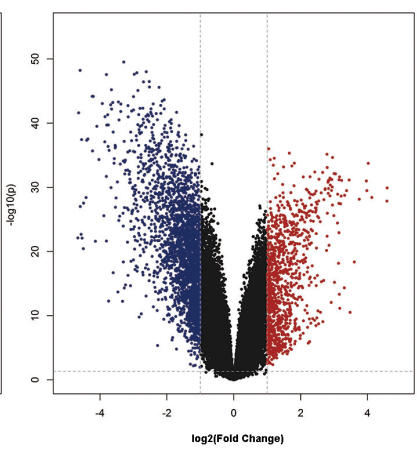

$\mathrm{F}$

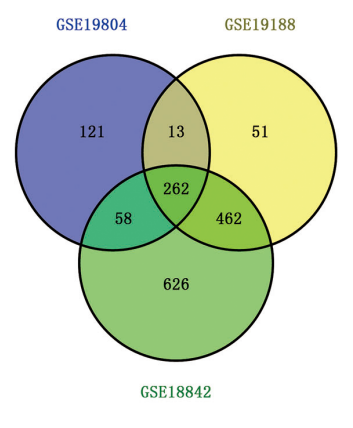

C

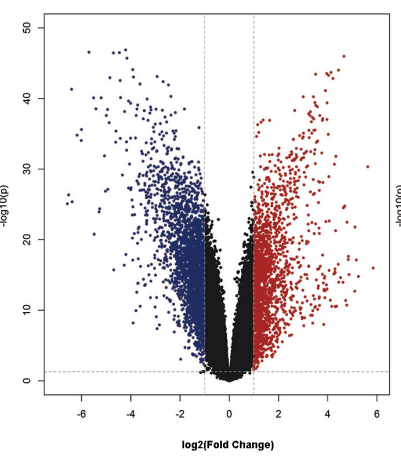

G

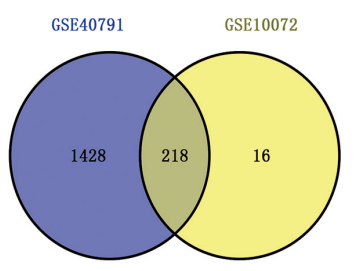

D

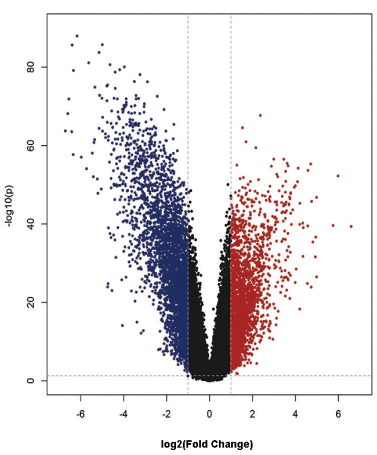

$\mathrm{H}$

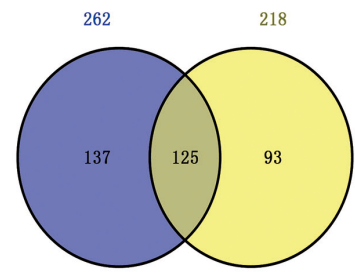

Figure 1: Identification of expression differences between tumor and NTL. A-E. Volcano plot of the differential mRNA expression analysis. X-axis: $\log 2$ fold change; Y-axis: - $\log 10$ (FDR P-value) for each probes; Vertical dotted lines: fold change $\geq 2$ or $\leq 2$; Horizontal dotted line: the significance cutoff $($ FDR P-value $=0.05)$. (A) There were 1,388 genes identified to be differentially expressed in GSE19804, including 454 up-regulated and 934 down-regulated genes. (B) 2,421 genes (788 up-regulated and 1,633 down-regulated genes) differentially expressed in GSE19188. (C) 3,168 genes (1,403 up-regulated and 1,765 down-regulated genes) differentially expressed in GSE18842. (D) 3,796 genes (1,646 up-regulated and 2,150 down-regulated genes) differentially expressed in GSE40791. (E) 666 genes (234 up-regulated and 432 down-regulated genes) differentially expressed in GSE18842. F-H. Overlap analysis between different datasets. (F) A total of 262 genes were significantly differentially expressed in the three NSCLC datasets. (G) 218 genes were overlapped in the two LUAD datasets. $(\mathrm{H})$ There were 125 overlapping genes significantly differentially expressed between tumor and NTL in all five datasets. 
genes which were specifically overexpressed in LUAD. As indicated in Figure 1F, a total of 262 genes were significantly differentially expressed in the three NSCLC datasets. 218 genes were overlapped in the two LUAD datasets as shown in Figure 1G. After further screening by overlapping these two subsets of genes, 125 genes were identified that could be exclusive to LUAD carcinogenesis (Figure 1H, Supplementary Table S1).

\section{Function enrichment of differentially expressed genes}

To determine biological functions of the 125 DEGs, Gene Ontology (GO) analysis was performed. In terms of the three different domains of GO, genes associated with biological processes were mainly involved in cell cycle and mitosis, 5 of the top 10 enriched categories of molecular function were related to peptidase activity or kinase activity, while spindle and chromosome were the most enriched cellular components (Figure 2A, Supplementary Table S2). We further investigated the functional implication of these DEGs in the development of LUAD by Kyoto Encyclopedia of Genes and Genomes (KEGG) analysis. The results demonstrated that DEGs were enriched in six KEGG pathways. Cell cycle was the most significant one $\left(\mathrm{P}=1.52 \times 10^{-6}\right)$, followed by ECM-receptor interaction $\left(\mathrm{P}=9.62 \times 10^{-6}\right), \mathrm{p} 53$ signaling pathway $\left(\mathrm{P}=3.66 \times 10^{-4}\right)$ (Figure $2 \mathrm{~B}$, Supplementary Table S3). Most of enriched functional catalogues and pathways have been found to be closely associated with the incidence and development of cancer, which emphasized an implication of the DEGs in LUAD. We also found that several genes were repeatedly involved in cell cycle and mitosis, including MAD2L1 and CDK1.

\section{CDK1 and MAD2L1 were overexpressed in LUAD}

CDK1 and MAD2L1 were selected for further study due to their known role in regulating tumor cell cycle and mitosis. The increased expression of CDK1 and MAD2L1 in LUAD was identified in five discovering datasets (three NSCLC datasets and two LUAD datasets) (Figure 3A and $3 \mathrm{~B}$ ). Furthermore, we validated their over-expression by using the Cancer Genome Atlas (TCGA) database. A total of 349 LUAD tissues and 58 NTL tissue samples were selected. The expression levels of the two selected genes were in line with those of our training cohorts, with significant differences between tumor and NTL (Figure 4A and 4B). All these results suggested that overexpression of CDK1 and MAD2L1 was a common feature for LUAD. We also found that CDK1 and MAD2L1 expression was elevated in patients with a higher pathological stage (Figure 4C and 4D).

\section{Associations of CDK1 and MAD2L1 expression with clinicopathological variables}

Clinicopathological characteristics of the LUAD patients are listed in Table 1. CDK1 expression was remarkably positively associated with gender $(\mathrm{P}=0.008)$, smoking history $(\mathrm{P}<0.001)$, pathologic $\mathrm{T}$ stage $(\mathrm{P}=$
A

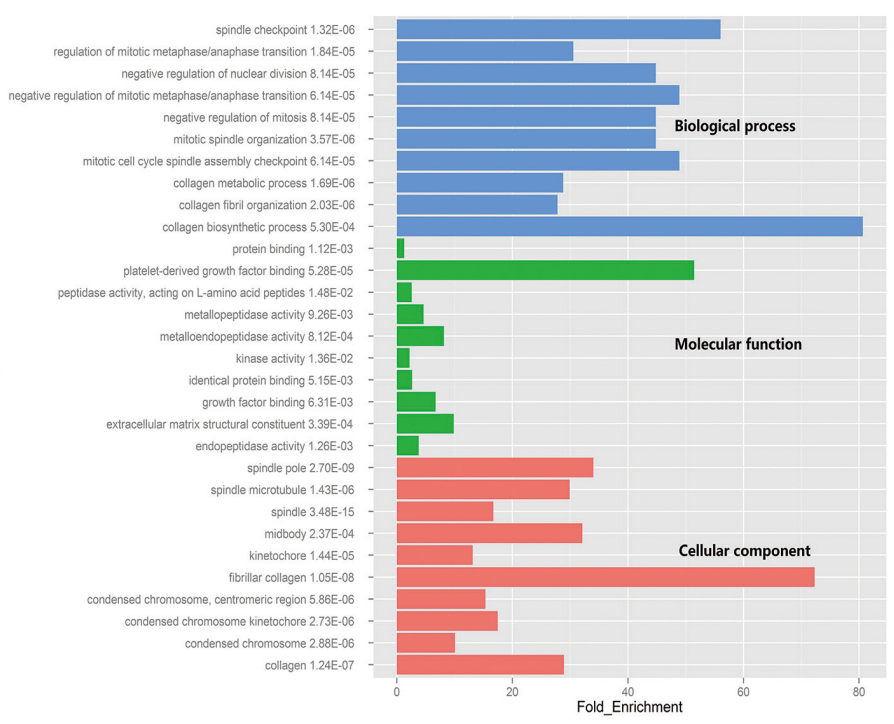

B

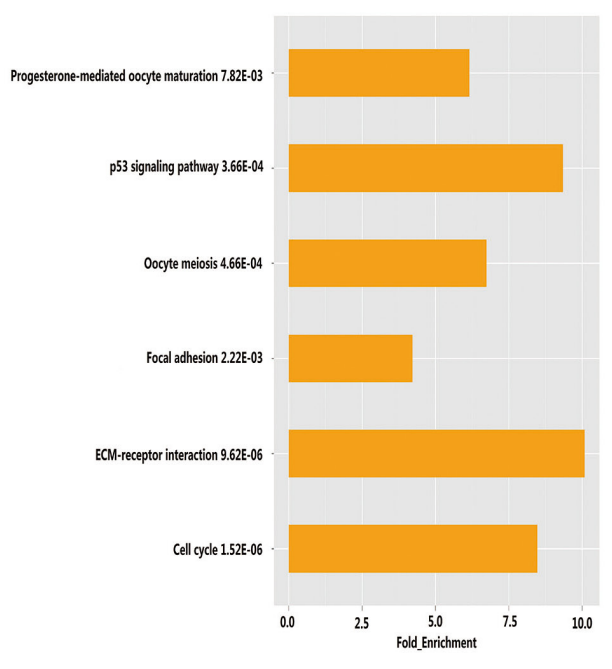

Figure 2: GO and pathway analysis of significant differentially expressed genes. A. The top ten significantly enriched GO categories were calculated. Blue: Biological process; Green: Molecular function; Red: Cellular component. B. Gene networks identified through KEGG analysis of the differentially expressed genes. 

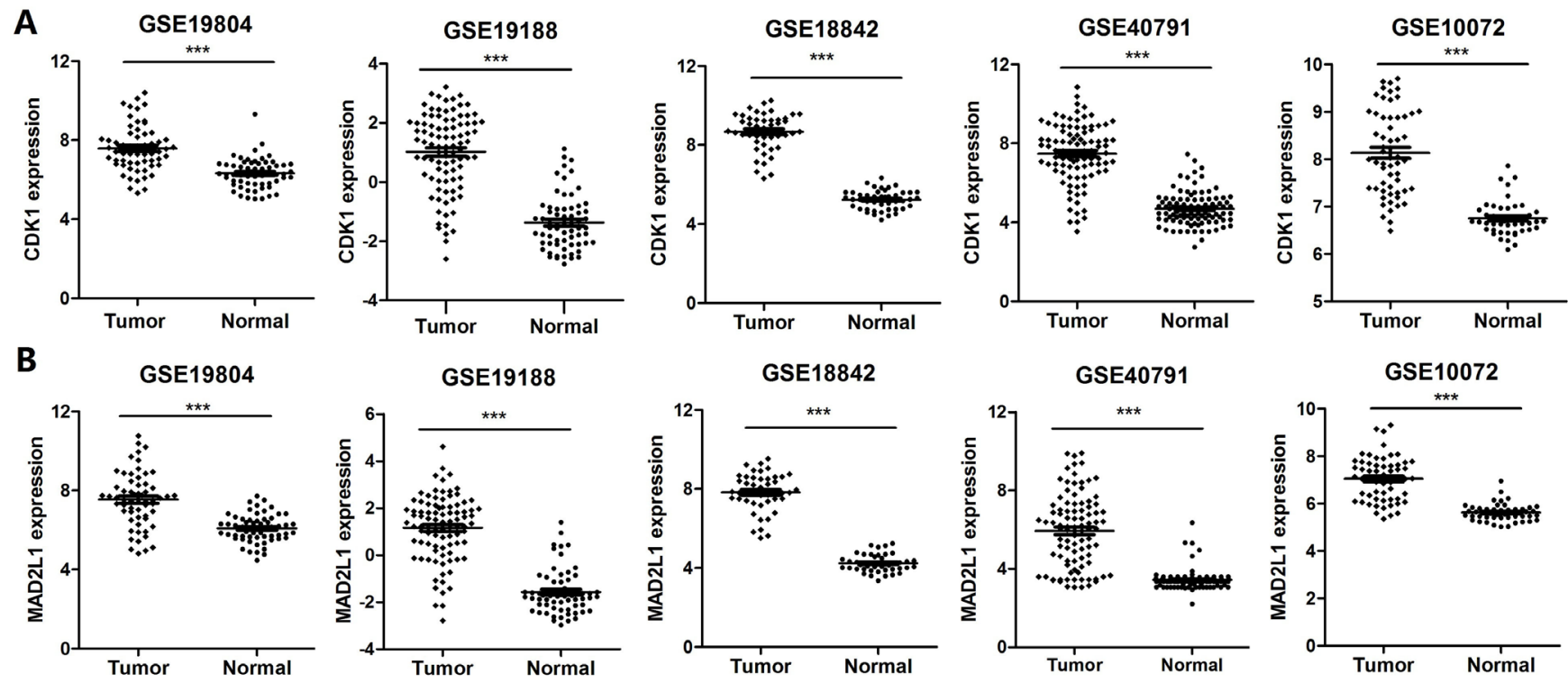

Figure 3: Identification of the differentially expressed genes. A. Identification of mRNA expression of CDK1 in five datasets, respectively. B. Identification of mRNA expression of MAD2L1 in five datasets, respectively. *** corresponds to $\mathrm{P}<0.001 ; * * \mathrm{P}<0.01$ and $* \mathrm{P}<0.05$.
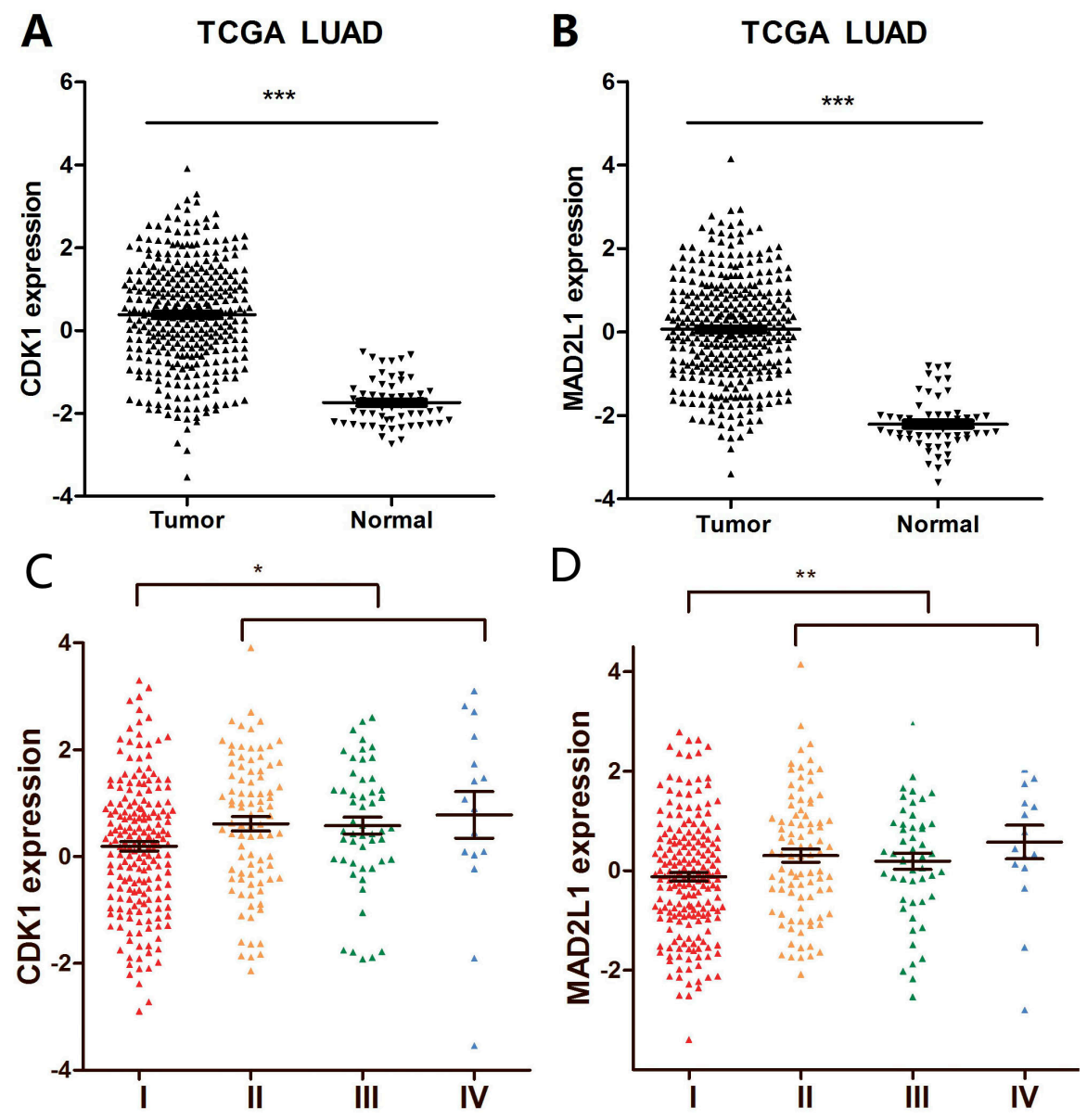

Figure 4: Validation of the differentially expressed genes. A. Validation of mRNA expression of CDK1 in TCGA datasets. B. Validation of mRNA expression of MAD2L1 in TCGA datasets. C. Gene expression of CDK1 in LUAD patients according to clinical stage. D. Gene expression of MAD2L1 in LUAD patients according to clinical stage. *** corresponds to $\mathrm{P}<0.001 ; * * \mathrm{P}<0.01$ and $* \mathrm{P}<0.05$. 
Table 1: Clinical characteristics and correlations with the expression of CDK1 and MAD2L1

\begin{tabular}{|c|c|c|c|c|c|c|c|}
\hline \multirow[b]{2}{*}{ Characteristic } & \multirow[b]{2}{*}{$n=349$} & \multicolumn{3}{|c|}{ CDK1 } & \multicolumn{3}{|c|}{ MAD2L1 } \\
\hline & & $\begin{array}{c}\text { Low } \\
(n=174)\end{array}$ & $\begin{array}{c}\text { High } \\
(\mathrm{n}=175)\end{array}$ & P value & $\begin{array}{c}\text { Low } \\
(n=174)\end{array}$ & $\begin{array}{c}\text { High } \\
(\mathrm{n}=175)\end{array}$ & P value \\
\hline \multicolumn{8}{|l|}{ Age (years) } \\
\hline$<65$ & 159 & 74 & 85 & 0.257 & 71 & 88 & 0.075 \\
\hline$\geq 65$ & 190 & 100 & 90 & & 103 & 87 & \\
\hline \multicolumn{8}{|l|}{ Gender } \\
\hline Female & 190 & 107 & 83 & 0.008 & 111 & 79 & $<0.001$ \\
\hline Male & 159 & 67 & 92 & & 63 & 96 & \\
\hline \multicolumn{8}{|l|}{ Smoking history } \\
\hline Current smoker & 80 & 28 & 52 & $<0.001$ & 27 & 53 & $<0.001$ \\
\hline $\begin{array}{l}\text { Current reformed smoker } \\
\text { for } \leq 15 \text { years }\end{array}$ & 115 & 48 & 67 & & 51 & 64 & \\
\hline $\begin{array}{l}\text { Current reformed smoker } \\
\text { for }>15 \text { years }\end{array}$ & 85 & 57 & 28 & & 56 & 29 & \\
\hline Never-smoker & 53 & 33 & 20 & & 34 & 19 & \\
\hline \multicolumn{8}{|l|}{ Clinical stage } \\
\hline I \& II & 281 & 146 & 135 & 0.111 & 149 & 132 & 0.016 \\
\hline III \& IV & 68 & 28 & 40 & & 25 & 43 & \\
\hline \multicolumn{8}{|l|}{ New tumor event } \\
\hline YES & 99 & 40 & 59 & 0.26 & 41 & 58 & 0.047 \\
\hline $\mathrm{NO}$ & 250 & 134 & 116 & & 133 & 117 & \\
\hline \multicolumn{8}{|l|}{ Pathologic T stage } \\
\hline $\mathrm{T} 1$ & 127 & 80 & 47 & 0.001 & 81 & 46 & 0.001 \\
\hline $\mathrm{T} 2$ & 180 & 77 & 103 & & 77 & 103 & \\
\hline $\mathrm{T} 3+\mathrm{T} 4$ & 40 & 16 & 24 & & 16 & 24 & \\
\hline \multicolumn{8}{|l|}{ Therapy outcome } \\
\hline $\mathrm{CR}+\mathrm{PR}$ & 145 & 83 & 62 & 0.015 & 77 & 68 & 0.098 \\
\hline $\mathrm{SD}+\mathrm{PD}$ & 86 & 35 & 51 & & 36 & 50 & \\
\hline
\end{tabular}

$0.001)$ and therapy outcome $(\mathrm{P}=0.015)$. No significant difference of CDK1 mRNA levels was found in patients with different age, clinical stage and new tumor event (P >0.05). As Table 1 showed, the elevated MAD2L1 expression was significantly correlated with gender $(\mathrm{P}<$ $0.001)$, smoking history $(\mathrm{P}<0.001)$, clinical stage $(\mathrm{P}=$ $0.016)$ and pathologic $\mathrm{T}$ stage $(\mathrm{P}=0.001)$.

\section{Associations of CDK1 and MAD2L1 expression with overall and relapse-free survival}

We next asked whether CDK1 and MAD2L1 expression will influence clinical outcomes of LUAD patients. As shown in Figure 5A and Figure 5B, CDK1 expression was significantly related with $\mathrm{OS}(\mathrm{P}=0.02)$ and RFS $(\mathrm{P}=0.02)$ of LUAD patients. The median OS in CDK1 low expression group is 59.7 months, in CDK1 high expression group is 43.1 months. The median RFS in CDK1 low expression group is 68.2 months, in CDK1 high expression group is 26.9 months. Those figures meant that higher CDK1 expression indicated poorer prognosis and earlier recurrence. Similarly, elevated expression of MAD2L1 was both remarkably associated with reduced survival $(\mathrm{P}=0.01$; Figure $5 \mathrm{C})$ and increased risk of recurrence $(\mathrm{P}=0.01$; Figure $5 \mathrm{D})$. The median $\mathrm{OS}$ in low and high group of MAD2L1 is 59.7 months and 
43.1 months. The median RFS in low and high group of MAD2L1 is 73.9 months and 25.7 months. In short, the overexpressed CDK1 and MAD2L1 have potentials to serve as prognostic biomarkers for prediction of LUAD recurrence and survival.

\section{DISCUSSION}

High-throughput analyses are used to determine gene expression signatures for improved accuracy of prognosis [10]. In order to identify potential biomarkers for LUAD prognosis and therapy, we integrated expression profiles of 668 lung cancer patients in five datasets from the Gene Expression Omnibus (GEO) and identified a panel of differentially expressed genes (DEGs). Function enrichment analysis highlighted that these genes were closely related to the carcinogenesis of LUAD, such as cell cycle, ECM-receptor interaction and p53 signaling pathway. CDK1 and MAD2L1, two critical mitotic checkpoint genes, were selected for further study. Elevated expression of CDK1 and MAD2L1 was validated via an independent LUAD cohort. KaplanMeier analysis revealed that CDK1 and MAD2L1 correlated with both overall survival (OS) and relapsefree survival (RFS).

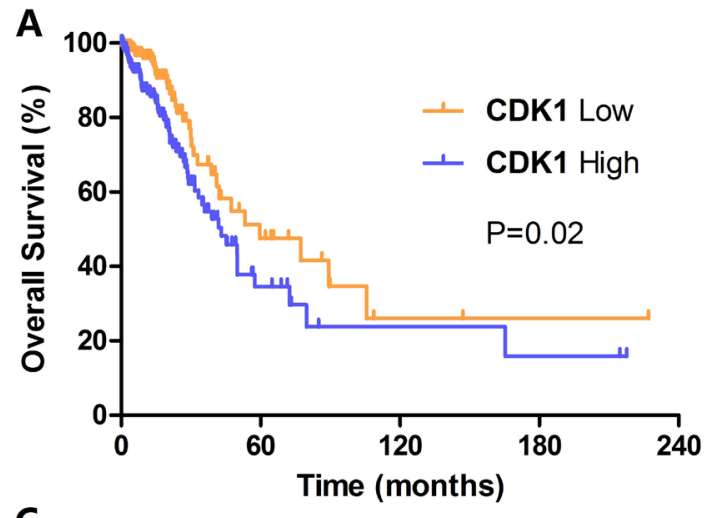

C

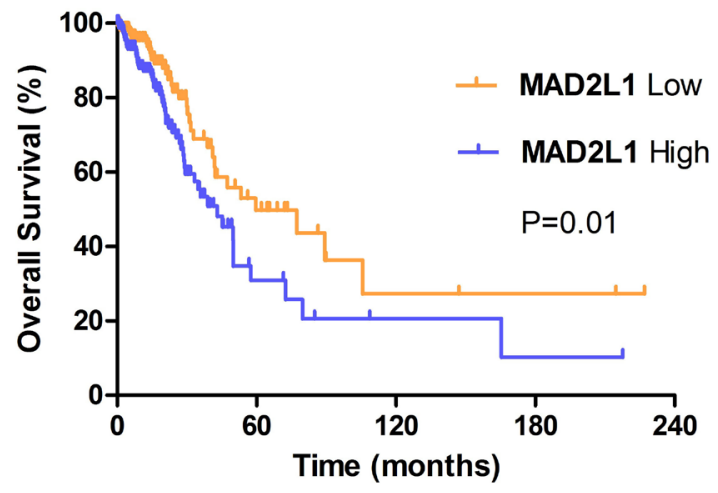

The cell cycle is an evolutionarily conserved process necessary for mammalian cell growth and development. Loss of normal cell-cycle control is a hallmark of human cancer [11]. Recently, many therapeutic strategies have been proposed for targeting the cell cycle in cancer. Chromosomal instability correlates with poor prognosis in multiple solid tumors, indicating that increasing genetic diversity contributes to changed tumor survival and chemoresistance [12]. At present, several cell cycle related genes have been reported to be involved in lung cancer initiation and development. Ding et al. [13] discovered that CCNB1 was a biomarker for the prognosis of ER+ breast cancer and monitoring of hormone therapy efficacy. Qian et al. [14] found that overexpression of CCNB2 protein was associated with clinical progression and poor prognosis in NSCLC. Another research showed that ISL1 promoted tumor cell proliferation and tumorigenesis. ISL1 serves as a novel regulator for the expression of CCNB1, CCNB2 and C-MYC, which plays significant roles in gastric cancer progression and development [15].

Cyclin-dependent kinases (CDKs) are important cell cycle-regulating proteins [16]. CDK1 is essential for cell cycle progression and proliferation, and the dysregulation of CDK1 activity was a common event in a variety of tumors [17-19]. The results in this study were similar to those published by others to date, which

B

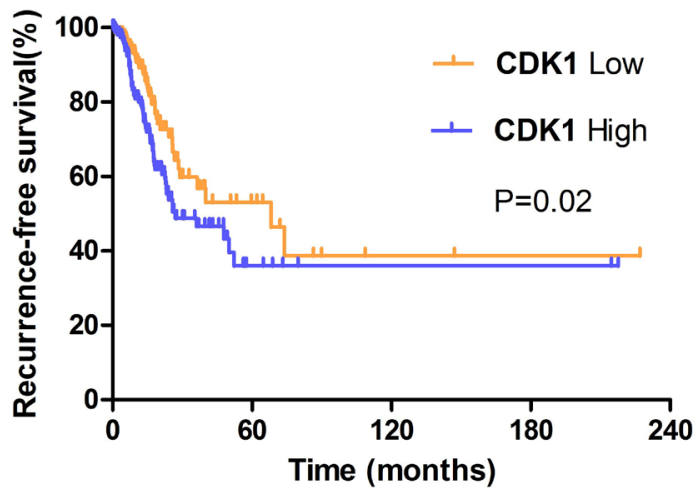

D

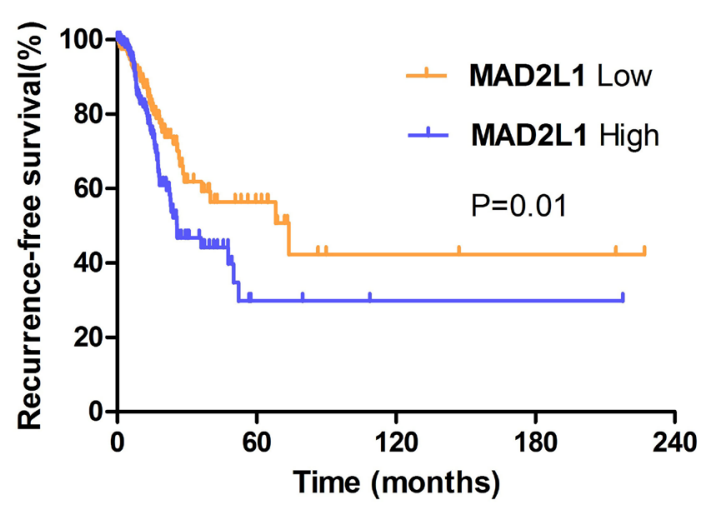

Figure 5: Kaplan-Meier survival curves by different levels of CDK1 and MAD2L1 expression in 349 LUAD patients. A. Overall survival (OS) by low and high CDK1 expression; B. Relapse-free survival (RFS) by low and high CDK1 expression; C. Overall survival (OS) by low and high MAD2L1 expression; D. Relapse-free survival (RFS) by low and high MAD2L1 expression. 
showed higher CDK1 expression and activity in prostate cancer, colorectal cancer and epithelial ovarian cancer [20-22]. Another potential prognostic factor for LUAD is MAD2L1. MAD2L1 is required in mitosis when chromosomes are unattached to the mitotic spindle that maintains chromosomal segregation, and is involved in the spindle checkpoint during mitosis [23-25]. Dysregulation of MAD2L1 is associated with chromosomal instability and substantial aneuploidy which frequently occur in cancer [26].

In conclusion, the present study has shown that CDK1 and MAD2L1 are overexpressed in LUAD tissues and that its up-regulation may be indicative of poor survival rates and a higher risk for cancer recurrence, and it could have a potential role as a prognostic marker in LUAD patients. Functional researches are necessary to reveal the molecular mechanisms of CDK1 and MAD2L1 in LUAD and their role in prognosis and therapeutic target.

\section{MATERIALS AND METHODS}

All data analysis were performed using R (http:// www.r-project.org/, version 2.15.0) and Bioconductor [27].

\section{Lung cancer patient datasets}

Training datasets (GSE19804, GSE19188, GSE18842, GSE40791, GSE10072) based on the Affymetrix platform (Affymetrix HG-U133 Plus 2.0 array and HG-U133A array) and corresponding clinical information of lung cancer patients were retrieved from Gene Expression Omnibus (http://www.ncbi.nlm.nih. gov/geo). Three NSCLC genome-wide expression profiles were extracted from the following three datasets: GSE19804, which includes 60 paired tumor and normal tissues, GSE19188, including 91 tumor and 65 adjacent normal lung tissues, and GSE18842, which includes 46 tumors and 45 controls. Two LUAD genome-wide expression profiles were extracted from GSE40791 and GSE10072, with the former including 94 tumor and 100 non-tumor tissues and the latter containing 58 tumor and 49 non-tumor tissues.

Validation datasets were downloaded from the Cancer Genome Atlas (TCGA) data portal (http://tcga-data.nci. nih.gov). We selected 349 tumor and 58 NTL samples, with both mRNA expression data and clinical features information available for performing the correlation analysis and survival analysis. Detailed clinical information of patients used in this study was shown in Table 1.

\section{Global gene expression analysis}

Raw microarray data files (.CEL files) of the five patient datasets were downloaded from the GEO database.
Background correction and quartile normalization were performed using the Robust Multichip Average (RMA) algorithm by the R package Affy [28, 29]. After that, the Linear Models for Microarray Data (LIMMA) package in $\mathrm{R}$ was used to calculate the probability of probes being differentially expressed between cases and controls. The fold change (FC) and its logarithm value (log FC) were also determined. Corrected P-value $<0.05$ and absolute fold change $>2$ were used to identify significantly differential expressed mRNAs. The heat map, locusby-locus volcano plot and venn diagram of significant differentially expressed mRNA were performed by gplots, lattice, and venn diagram packages in $R$, respectively [30].

\section{Functional enrichment analysis}

The Database for Annotation, Visualization and Integrated Discovery (DAVID) bioinformatics resource consists of an integrated biological knowledge base and analytic tools aimed at systematically extracting biological meaning from large gene or protein lists [31, 32]. In the present study, DAVID was applied to conduct Kyoto encyclopedia of genes and genomes (KEGG) pathway and gene ontology (GO) enrichment analyses for the identified target genes. KEGG is a knowledge base for systematic analysis of gene functions. GO analysis predicts the function of the target genes in three aspects, including biological processes, cellular components and molecular function. Functional annotation with a $\mathrm{P}$-value $<0.05$ and an enrichment score $>2.0$ were considered statistically significant.

\section{Statistical analysis}

Statistical analyses were performed with SPSS version 18.0 and GraphPad Prism 5.0 software. Single comparisons between two groups were determined by Student's t-test. Survival analysis was carried out according to Kaplan-Meier analysis and Log-rank test. Two types of survival outcomes were included in survival analysis: overall survival (OS), defined as the time between the date of surgery and date of death or last follow-up, and relapse-free survival (RFS), defined as the period from surgery to recurrence or last followup. P-values less than 0.05 were considered statistically significant.

\section{ACKNOWLEDGMENTS}

We thank all supporting funds from the National High-tech R\&D Program of China (863 Program) (2012AA02A517), National Natural Science Foundation of China (81373490, 81573508, 81573463), and Hunan Provincial Science and Technology Plan of China (2015TP1043). 


\section{CONFLICTS OF INTEREST}

There are no conflicts of interest.

\section{REFERENCES}

1. Siegel RL, Miller KD, Jemal A. Cancer Statistics, 2015. Ca-a Cancer Journal for Clinicians. 2015; 65: 5-29. doi: $10.3322 /$ caac. 21254 .

2. Zappa C, Mousa SA. Non-small cell lung cancer: current treatment and future advances. Translational Lung Cancer Research. 2016; 5: 288-300. doi: 10.21037/tlcr.2016.06.07.

3. Andersson E, Arvidsson Y, Sward C, Hofving T, Wangberg B, Kristiansson E, Nilsson O. Expression profiling of small intestinal neuroendocrine tumors identifies subgroups with clinical relevance, prognostic markers and therapeutic targets. Modern Pathology. 2016; 29: 616-29. doi: 10.1038/ modpathol.2016.48.

4. Chen F, Xiang CX, Zhou Y, Ao XS, Zhou DQ, Peng P, Zhang HQ, Liu HD, Huang X. Gene expression profile for predicting survival of patients with meningioma. International Journal of Oncology. 2015; 46: 791-7. doi: 10.3892/ijo.2014.2779.

5. Lu Y, Lemon W, Liu PY, Yi YJ, Morrison C, Yang P, Sun ZF, Szoke J, Gerald WL, Watson M, Govindan R, You M. A gene expression signature predicts survival of patients with stage I non-small cell lung cancer. Plos Medicine. 2006; 3: 2229-43. doi: ARTN e467 10.1371/journal.pmed.0030467.

6. Raponi M, Zhang Y, Yu J, Chen G, Lee G, Taylor JMG, MacDonald J, Thomas D, Moskaluk C, Wang YX, Beer DG. Gene expression signatures for predicting prognosis of squamous cell and adenocarcinomas of the lung. Cancer Research. 2006; 66: 7466-72. doi: 10.1158/0008-5472. CAN-06-1191.

7. Ioannidis JPA, Allison DB, Ball CA, Coulibaly I, Cui XQ, Culhane AC, Falchi M, Furlanello C, Game L, Jurman G, Mangion J, Mehta T, Nitzberg M, et al. Repeatability of published microarray gene expression analyses. Nature Genetics. 2009; 41: 149-55. doi: 10.1038/ng.295.

8. Sun ZF, Wigle DA, Yang P. Non-overlapping and non-celltype-specific gene expression signatures predict lung cancer survival. Journal of Clinical Oncology. 2008; 26: 877-83. doi: 10.1200/Jco.2007.13.1516.

9. Lohr M, Kollmann C, Freis E, Hellwig B, Hengstler JG, Ickstadt K, Rahnenfuhrer J. Optimal Strategies for Sequential Validation of Significant Features from HighDimensional Genomic Data. Journal of Toxicology and Environmental Health-Part a-Current Issues. 2012; 75: 44760. doi: 10.1080/15287394.2012.674912.

10. Ko JH, Ko EA, Gu W, Lim I, Bang H, Zhou T. Expression profiling of ion channel genes predicts clinical outcome in breast cancer. Molecular Cancer. 2013; 12. doi: Artn 106 10.1186/1476-4598-12-106.

11. Dominguez-Brauer C, Thu KL, Mason JM, Blaser H, Bray MR, Mak TW. Targeting Mitosis in Cancer: Emerging
Strategies. Molecular Cell. 2015; 60: 524-36. doi: 10.1016/j. molcel.2015.11.006.

12. Visconti R, Della Monica R, Grieco D. Cell cycle checkpoint in cancer: a therapeutically targetable doubleedged sword. Journal of Experimental \& Clinical Cancer Research. 2016; 35. doi: Artn 15310.1186/ S13046-016-0433-9.

13. Ding K, Li WQ, Zou ZQ, Zou XZ, Wang CR. CCNB1 is a prognostic biomarker for ER plus breast cancer. Medical Hypotheses. 2014; 83: 359-64. doi: 10.1016/j. mehy.2014.06.013.

14. Qian XT, Song XK, He Y, Yang ZY, Sun T, Wang J, Zhu GQ, Xing WH, You CX. CCNB2 overexpression is a poor prognostic biomarker in Chinese NSCLC patients. Biomedicine \& Pharmacotherapy. 2015; 74: 222-7. doi: 10.1016/j.biopha.2015.08.004.

15. Shi Q, Wang WP, Jia ZQ, Chen P, Ma KT, Zhou CY. ISL1, a novel regulator of CCNB1, CCNB2 and c-MYC genes, promotes gastric cancer cell proliferation and tumor growth. Oncotarget. 2016; 7: 36489-500. doi: 10.18632/ oncotarget.9269.

16. Malumbres M, Barbacid M. Cell cycle, CDKs and cancer: a changing paradigm. Nature Reviews Cancer. 2009; 9: 15366. doi: $10.1038 / \mathrm{nrc} 2602$.

17. Santamaria D, Barriere C, Cerqueira A, Hunt S, Tardy C, Newton K, Caceres JF, Dubus P, Malumbres M, Barbacid M. Cdk1 is sufficient to drive the mammalian cell cycle. Nature. 2007; 448: 811-U8. doi: 10.1038/nature06046.

18. Barascu A, Besson P, Le Floch O, Bougnoux P, Jourdan ML. CDK1-cyclin B1 mediates the inhibition of proliferation induced by omega-3 fatty acids in MDA-MB-231 breast cancer cells. International Journal of Biochemistry \& Cell Biology. 2006; 38: 196-208. doi: 10.1016/j. biocel.2005.08.015.

19. Zeestraten ECM, Maak M, Shibayama M, Schuster T, Nitsche U, Matsushima T, Nakayama S, Gohda K, Friess H, van de Velde CJH, Ishihara H, Rosenberg R, Kuppen PJK, et al. Specific activity of cyclin-dependent kinase I is a new potential predictor of tumour recurrence in stage II colon cancer (vol 106, pg 133, 2012). British Journal of Cancer. 2012; 106: 792-. doi: 10.1038/bjc.2012.38.

20. Willder JM, Heng SJ, McCall P, Adams CE, Tannahill C, Fyffe G, Seywright M, Horgan PG, Leung HY, Underwood MA, Edwards J. Androgen receptor phosphorylation at serine 515 by $\mathrm{Cdk} 1$ predicts biochemical relapse in prostate cancer patients. British Journal of Cancer. 2013; 108: 13948. doi: 10.1038/bjc.2012.480.

21. Sung WW, Lin YM, Wu PR, Yen HH, Lai HW, Su TC, Huang RH, Wen CK, Chen CY, Chen CJ, Yeh KT. High nuclear/cytoplasmic ratio of Cdk1 expression predicts poor prognosis in colorectal cancer patients. BMC Cancer. 2014; 14. doi: Artn 951 10.1186/1471-2407-14-951.

22. Yang W, Cho H, Shin HY, Chung JY, Kang ES, Lee EJ, Kim JH. Accumulation of cytoplasmic Cdk1 is associated with cancer growth and survival rate in epithelial ovarian 
cancer. Oncotarget. 2016; 7: 49481-97. doi: 10.18632/ oncotarget. 10373.

23. Pulverer B. Spindle checkpoint protein links Rb pathway to aneuploidy. Nature Cell Biology. 2004; 6: 806-. doi: 10.1038/ncb0904-806.

24. Wang ZW, Katsaros D, Shen Y, Fu YY, Canuto EM, Benedetto C, Lu LG, Chu WM, Risch HA, Yu H. Biological and Clinical Significance of MAD2L1 and BUB1, Genes Frequently Appearing in Expression Signatures for Breast Cancer Prognosis. PLoS One. 2015; 10. doi: ARTN e0136246 10.1371/journal.pone.0136246.

25. Guo YL, Zhang XM, Yang M, Miao XP, Shi YK, Yao JR, Tan W, Sun T, Zhao D, Yu DK, Liu JN, Lin DX. Functional evaluation of missense variations in the human MAD1L1 and MAD2L1 genes and their impact on susceptibility to lung cancer. Journal of Medical Genetics. 2010; 47: 616-22. doi: 10.1136/jmg.2009.074252.

26. Li Y, Benezra R. Identification of a human mitotic checkpoint gene: hsMAD2. Science. 1996; 274: 246-8.

27. Gentleman RC, Carey VJ, Bates DM, Bolstad B, Dettling M, Dudoit S, Ellis B, Gautier L, Ge YC, Gentry J, Hornik K, Hothorn T, Huber W, et al. Bioconductor: open software development for computational biology and bioinformatics. Genome Biology. 2004; 5. doi: Artn R80 10.1186/ Gb-2004-5-10-R80.
28. Irizarry RA, Bolstad BM, Collin F, Cope LM, Hobbs B, Speed TP. Summaries of affymetrix GeneChip probe level data. Nucleic Acids Research. 2003; 31. doi: ARTN e15 10.1093/nar/gng015.

29. Irizarry RA, Hobbs B, Collin F, Beazer-Barclay YD, Antonellis KJ, Scherf U, Speed TP. Exploration, normalization, and summaries of high density oligonucleotide array probe level data. Biostatistics. 2003; 4: 249-64. doi: 10.1093/biostatistics/4.2.249.

30. Wang Y, Qian CY, Li XP, Zhang Y, He H, Wang J, Chen J, Cui JJ, Liu R, Zhou H, Xiao L, Xu XJ, Zheng Y, et al. Genome-scale long noncoding RNA expression pattern in squamous cell lung cancer. Scientific Reports. 2015; 5. doi: Artn 11671 10.1038/Srep11671.

31. Huang DW, Sherman BT, Lempicki RA. Bioinformatics enrichment tools: paths toward the comprehensive functional analysis of large gene lists. Nucleic Acids Research. 2009; 37: 1-13. doi: 10.1093/nar/gkn923.

32. Liu R, Guo CX, Zhou HH. Network-based approach to identify prognostic biomarkers for estrogen receptorpositive breast cancer treatment with tamoxifen. Cancer Biology \& Therapy. 2015; 16: 317-24. doi: 10.1080/15384047.2014.1002360. 